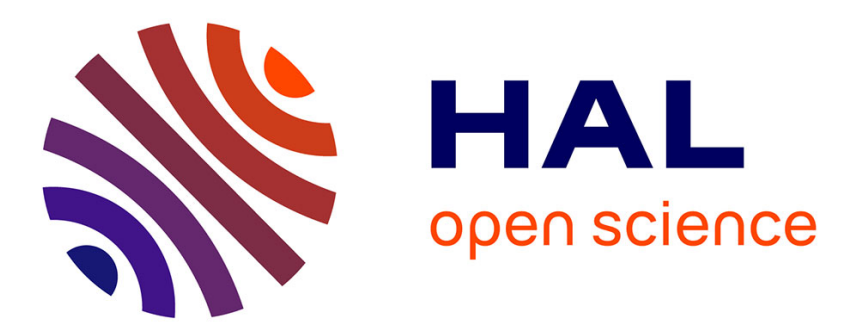

\title{
AFM and low-pressure argon adsorption analysis of geometrical properties of phyllosilicates
}

Malak Sayed-Hassan, Frédéric Villiéras, Fabien Gaboriaud, Angelina

Razafitianamaharavo

\section{To cite this version:}

Malak Sayed-Hassan, Frédéric Villiéras, Fabien Gaboriaud, Angelina Razafitianamaharavo. AFM and low-pressure argon adsorption analysis of geometrical properties of phyllosilicates. Journal of Colloid and Interface Science, 2006, 296, pp.614-623. 10.1016/j.jcis.2005.09.028 hal-00086907

\section{HAL Id: hal-00086907 https://hal.science/hal-00086907}

Submitted on 20 Jul 2006

HAL is a multi-disciplinary open access archive for the deposit and dissemination of scientific research documents, whether they are published or not. The documents may come from teaching and research institutions in France or abroad, or from public or private research centers.
L'archive ouverte pluridisciplinaire HAL, est destinée au dépôt et à la diffusion de documents scientifiques de niveau recherche, publiés ou non, émanant des établissements d'enseignement et de recherche français ou étrangers, des laboratoires publics ou privés. 


\section{AFM and low-pressure argon adsorption analysis of geometrical properties of phyllosilicates}

Malak Sayed Hassan ${ }^{1, *}$, Frédéric Villieras ${ }^{1}$, Fabien Gaboriaud ${ }^{2}$, Angelina Razafitianamaharavo ${ }^{1}$

1: LEM: Laboratoire Environnement et Minéralurgie, UMR 7569 INPL\&CNRS, Ecole Nationale Supérieure de Géologie, BP 40, 54501 Vandoeuvre-lès-Nancy, France

2: LCPME: Laboratoire de Chimie Physique et Microbiologie pour l'Environnement, UMR 7564 CNRS\&Université Henri Poincaré-Nancy 1, 405 rue de Vandoeuvre, 54600 Villers-lèsNancy, France

\section{* Corresponding author}

E-mail: malak.sayed-hassan@ensg.inpl-nancy.fr 


\begin{abstract}
The distribution of edge and basal surface areas of phyllosilicate particles is an essential parameter for understanding the interaction mechanisms at solid/gas or solid/liquid interfaces. Among the techniques proposed to determine the geometrical heterogeneities of flat solids, low pressure argon adsorption and AFM analysis are the most promising ones to derive the weightaveraged values of specific surface areas. A series of publications [1,2] have been recently dedicated to the combination of both methods showing the correlation between the two approaches. As the obtention of a large set of high resolution AFM images is time consuming it is necessary to test the ability of AFM routine analysis to derive systematically and statistically surface areas and aspect ratio, with all possible experimental and instrumental artefacts.

In the present study, the expected agreement was found between AFM and argon adsorption determination for total, basal and edge specific surface areas of non swelling clay minerals, except for one kaolinite, which is very heterogeneous in sizes. In addition, it was observed that for a given sample, individual particles present similar shapes, whatever their size, allowing to derive statistical relationship between AFM basal and total surface areas. On the basis of the obtained results, recommendations are given to derive accurate edge, basal and total specific surface areas of phyllosilicates by combining conventional gas adsorption (nitrogen BET) and routine AFM techniques.
\end{abstract}

Keywords: AFM, gas adsorption, surface heterogeneity, specific surface area, clay minerals 


\section{Introduction}

The particle shape is a fundamental feature of phyllosilicates, which depends on the physicochemical conditions of crystallization growth transport and deposition. In the case of kaolinites, a close relationship was established by Cases and coworkers between crystallinity, size and shape $[3,4]$. Phyllosilicates should then be considered as heterogeneous minerals with ${ }^{2}$ two types of surfaces, basal and edge (or lateral) surfaces (Figure 1), having different surface chemistry and properties. Considering particle sizes, many phyllosilicates are naturally found as finely divided particles (clay minerals) with non-negligible specific surface areas. As a consequence, surface properties of phyllosilicates can control porosity, permeability and exchange of contaminants and nutrients in soils and porous rocks. In addition, these minerals find numerous applications, taking advantage of their composition, specific surface area and/or lamellar particle shape. To determine the reactive surfaces of phyllosilicates [5] and understand interaction mechanisms at a molecular level, the distribution between basal and edge faces should then be determined.

Among the several techniques proposed for quantifying dimensions of particles, electron microscopy, such as Scanning Electron Microscopy (SEM) and Transmission Electron Microscopy (TEM), is the most widely used technique. These methods based on electron micrographs provide two-dimensional projection that can be further used to derive size, shape and morphological information of the basal faces. It is however sometime impossible to clearly differentiate between agglomerated particles and grains and sample preparation is sometimes very difficult and tedious [6]. Moreover, the determination of particle thickness is limited to the orientation of particles on SEM images and the accurate determination of the complete geometrical features for one particle is prevented from the bidimensional nature of these methods. 
Recently, the atomic force microscopy (AFM) has brought an alternative approach to characterize the morphology and the atomic surface or lattice structure of numerous minerals like oxides [2,7-10] or clay particles [1,11-13]. The great advantage of the AFM technique lies in the potentiality to assess the geometrical features of individual particles from the three dimensional AFM images. Unfortunately, this method requires important running time to obtain high resolution AFM images. Derivation of faces' surface areas and particle volumes become then a priori simple and particle shape analysis of phyllosilicates such as kaolinites was obtained by several authors. Recently, Zbick et al. [14] developed and discussed the AFM method to estimate the aspect ratio of kaolinite particles and compared his results with SEM estimations. Although the AFM approach appears promising for the quantification of basal and edge surface areas, literature results appear scattered, probably because of the distribution of the particle sizes and the low amount of analyzed particles. By using shadowed transmission electron microscopy, Lietard et al. [15] obtained accurate data on particle shape of kaolinite after the analysis of more than 4000 particles. When less particles are taken into account (between 400 and 700, [3]) the determination of geometrical properties from images is not accurate as this method favors the sampling of the smallest particles in the case of broad particle size distributions. An independent determination technique, less sensitive to the distribution of particle sizes, appears necessary to confirm AFM experimental and interpretation conditions that should be implemented to derive reliable information on geometrical properties of phyllosilicate particles.

Among the other experimental approaches that can be used to determine particle shape of phyllosilicates, Cases and coworkers proposed to take advantage of molecular interactions at solid/gas or solid/liquid interfaces [3,4,16-18]. In this case, the interaction between the surface and the molecules of the first monolayer depends on the adsorption energy of the different surface sites. It was then established by Cases et al. in 1896 [3] that the molecular probe technique is able to distinguish between adsorption on basal and edge faces by using cationic 
surfactants at the solid/liquid interface or argon at the solid/gas interface. In the case of argon adsorption at $77 \mathrm{~K}$, relevant information on particle shape were obtained for many phyllosilicates such as kaolinite, illite, saponite, montmorillonite and lamellar silica and oxy-hydroxides of iron and $\mathrm{Mn}[1,3,10,19,20,21,22,23]$. In addition, it was demonstrated that $\mathrm{N}_{2}$ probe cannot be used for the determination of basal and edge surface areas due to the polarizability (inducible quadrupolar momentum) of nitrogen molecules in the presence polar groups (mainly $\mathrm{OH}$ ) at the mineral surfaces $[3,18,19]$.

In the present study, we investigated the capability of AFM analysis to derive statistically the specific surface areas of clay minerals from routinely AFM measurements. Recent works suggest that the analysis of specific surface areas from AFM is not perturbed by the quality of the recorded images [2,10]. It is then interesting to record rapidly a large set of particle profiles by routine AFM experiments and to check for the accuracy of derived geometrical information based on results obtained by low pressure argon adsorption and DIS analysis of experimental data. Two non-swelling phyllosilicates were used: illite and kaolinite. Illite and kaolinite are common phyllosilicates that occur classically in sedimentary rocks as pure microcrystals or in interstratifications. In the case of kaolinite, reactive surfaces are mainly located on the edge faces due to the presence of hydrolysable $\mathrm{Si}-\mathrm{OH}$ and $\mathrm{Al}-\mathrm{OH}$ sites [24]. For illite, additional sites are present on basal surfaces, which correspond to the cations compensating structural charge defects. The use of one illite and two kaolinites allows then to check for the influence of surface chemistry and particle size distribution when deriving information on geometrical properties.

\section{MATERIALS AND METHODS}

\section{Solid preparation}

Illite was obtained from Le Puy orebody (France) and was previously studied by Bardot $[19,20]$. It was demonstrated that the exchange of surface cations by sodium or lithium is necessary to 
derive accurate argon specific surface area and aspect ratio [18,19,20]. In the present study, Bardot's results are used and described again to give interpretation keys for Na-illite sample. Kaolinite Kga2 was obtained from the Source Clays Repository of the Clays Minerals Society [25] and kaolinite Khg (High Gloss) is an industrial sample. Samples were purified and exchanged to $\mathrm{Na}$ by sedimentation of coarse particles, carbonate dissolution at $\mathrm{pH} 5,80^{\circ} \mathrm{C}$ (sodium acetate - acetic acid buffer), 3 exchanges in $\mathrm{NaCl} 1 \mathrm{M}$ followed by several washing and centrifugation cycles to remove excess ions.

The studied samples can be considered as not microporous as t-plot analysis performed on stepby step $\mathrm{N}_{2}$ adsorption isotherms shows that the samples do not present of significant amounts of micropores.

For microscopy experiments, samples were prepared as follows: $0.2 \mathrm{mg}$ sample material was mixed with $6 \mathrm{ml}$ water and a drop of $0.1 \mathrm{M} \mathrm{NaOH}$ and subsequently sonicated for 2 minutes to disperse the clay. The suspension drop was deposited on a glass slide and dried at $50{ }^{\circ} \mathrm{C}$ for SEM, AFM and on a copper grid for TEM.

\section{Electron microscopy}

The particle morphologies were investigated through scanning electron microscopy (SEM) and transmission electron microscopy (TEM) to check for the characteristic clay morphology. SEM images were obtained using a Hitachi S-2500 microscope equipped with a $\mathrm{LaB}_{6}$ electron source and TEM images were obtained with a Philips CM20 microscope operating at $200 \mathrm{KV}$.

\section{Low pressure argon adsorption at $77 \mathrm{~K}$}

Low-pressure isotherms of argon adsorption at 77K were recorded on a lab-built automatic quasiequilibrium volumetric set-up [16,17,26,27]. High purity argon (>99.995\%) was provided by 
Alphagaz (France). Around $1 \mathrm{~g}$ of sample was outgassed overnight at $110^{\circ} \mathrm{C}$ for kaolinite and $200^{\circ} \mathrm{C}$ for illite under a residual pressure of $10^{-4} \mathrm{~Pa}$. After outgassing, a slow, constant, and continuous flow of adsorbate was introduced into the adsorption cell through a micro-leak. The flow rate is constant, at least up to the BET domain, and can be adjusted by the pressure imposed before the leak. If the introduction rate is low enough, the measured pressures can be considered as quasi-equilibrium pressures (in the range of $10^{-3}, 3.10^{4} \mathrm{~Pa}$ ) $[26,27]$. From the recording of quasi-equilibrium pressure as a function of time, high-resolution adsorption isotherms were obtained with more than 2000 data points for the filling of the first monolayer. Due to the large number of experimental data points, the experimental derivative of the adsorbed quantity can be calculated as a function of the logarithm of relative pressure, $\ln (\mathrm{P} / \mathrm{Po})$, which corresponds to the free energy adsorption energy expressed in kT or RT units [16, 17, 27]. The derivative adsorption isotherm can then be considered as a fingerprint of the adsorption energy distribution for the considered gaseous probe.

The total derivative adsorption isotherm on a heterogeneous surface can be simulated by using concepts describing adsorption phenomena on heterogeneous surfaces [16-18]. The adsorption isotherm on a heterogeneous surface formed with different crystal faces can be written as:

$\theta_{t}=\sum_{i} X_{i} \theta_{i t}$

where $\theta_{t}$ is the total adsorption isotherm, and $\theta_{i t}$, are the adsorption isotherms on the different faces of the particle and $\mathrm{X}_{\mathrm{i}}$ its contribution to $\theta_{t}$. Each crystal face should be considered as heterogeneous and $\theta_{i t}$ writes:

$\theta_{i t}=\int_{\Omega} \theta_{i}(\varepsilon) \chi_{i}(\varepsilon) d \varepsilon$

Where $\varepsilon$ is the adsorption energy, $\Omega$ is the physical domain of $\varepsilon, \theta_{i}(\varepsilon)$ a 'local' theoretical adsorption isotherm and $\chi_{i}(\varepsilon)$ the dispersion of $\varepsilon$ on the $\mathrm{i}^{\text {th }}$ face or adsorption domain. It was shown by Villiéras et al. [16,17] that derivative forms of equations (1) and (2) can be used to 
model experimental derivative curves (derivative isotherm summation (DIS) procedure) by using theoretical local isotherms derived from the Langmuir (with Temkin improvement to take into account lateral interactions), BET (with Hill improvement to take into account lateral interactions) [16] and Dubinin-Astakhov [17] formalisms. In the present case, the BET-Hill derived formalism was used as the local $\theta_{i t}$ isotherm in equation (1). For each local isotherm, the following parameters are obtained: $\omega$, the lateral interactions between two neighboring adsorbed molecules, $\ln \left(\mathrm{P} / \mathrm{P}_{0}\right)$, the position of the peak and $\mathrm{Vm}$, the monolayer capacity.

\section{Atomic force microscopy}

AFM images were taken at ambient conditions in air using a commercial microscope (Thermomicroscope Explorer Ecu+, Veeco instrument S.A.S). To adhere single clay particles onto a glass substrate, the suspension drop was deposited on pre-heated clean glass slides and subsequently dried $\left(50^{\circ} \mathrm{C}, 1\right.$ h.). Different operation modes were used to achieve the AFM images of the different clay samples. For the illite and High Gloss clays, contact mode was used by using V-shaped cantilever (Ref MLCT-EXMT-BF, Veeco Instruments S.A.S). For the Kga2 sample, the images were collected by using non contact mode with etched microfabricated silicon tips (Ref 1650-00, Veeco Instruments S.A.S) at a resonance frequency of $245 \mathrm{kHz}$. AFM images were processed with the Scanning Probe Image Processor program (SPIP Version 2.3208, Image Metrology, Denmark) to correct baseline by using a fitted least mean square plane (Degree 1), to define the zero level from the height histogram and then to detect particles onto the three dimensional images.

The specific surface areas were calculated by using the procedure developed initially by Bickmore et al. [12] and recently extended by Jodin et al. [2]. Both methods cutout the particle in different horizontal slices with constant thickness and measured the corresponding perimeter $\mathrm{P}_{\mathrm{z}}$ 
[28], the area $A_{z}$, at the $z_{j}$ height level. From these results, two calculation methods were tested which consider either the whole particle or the top half of the particle. In the case of the whole particle (denoted $w$ ), the following equations were used to quantify the basal specific area BSA and edge specific area ESA of each i particle:

$$
\begin{gathered}
E S A_{i}^{w}=\frac{\sum_{j=Z_{0}}^{\text {Ztop }}\left(\left(Z_{j+1}-Z_{j}\right) \times P_{j}\right)}{\sum_{j=Z_{0}}^{\text {Zto }}\left(\left(Z_{j+1}-Z_{j}\right) \times A_{j} \times \rho\right)} \\
B S A_{i}{ }^{w}=\frac{A_{z_{0}}}{\sum_{j=Z_{0}}^{Z_{\text {top }}}\left(\left(Z_{j+1}-Z_{j}\right) \times A_{j} \times \rho\right)}
\end{gathered}
$$

where $\mathrm{z}_{\mathrm{top}}$ is the maximum height measured, $\mathrm{z}_{\mathrm{o}}$ the background level, and $\rho$ the density of clay $\left(\rho=2.62 \mathrm{~g} / \mathrm{cm}^{3}\right)$. In the case of the half particle method (denoted $\left.h\right)$, the specific surface areas were calculated according to:

$$
\begin{gathered}
E S A_{i}^{h}=\frac{\sum_{j=Z \text { top } / 2}^{\text {Ztop }}\left(\left(Z_{j+1}-Z_{j}\right) \times P_{j}\right)}{\sum_{j=Z \text { top } / 2}^{Z \text { top }}\left(\left(Z_{j+1}-Z_{j}\right) \times A_{j} \times \rho\right)} \\
B S A_{i}^{h}=\frac{A_{\text {Ztop } / 2}}{\sum_{j=Z \text { top } / 2}^{Z_{\text {top }}}\left(\left(Z_{j+1}-Z_{j}\right) \times A_{j} \times \rho\right)}
\end{gathered}
$$

At last, weight-averaged edge $<$ ESA $>$ and basal $<$ BSA $>$ specific surface areas were also calculated according to:

$$
<E S A>=\frac{\sum_{i}^{N} A_{e d g}^{i}}{\sum_{i}^{N} m^{i}}
$$


$<B S A>=\frac{\sum_{i}^{N} A_{b a s}^{i}}{\sum_{i}^{N} m^{i}}$

where $A_{e d g}^{i}$ and $A_{b a s}^{i}$ are the edge and basal area for each $i$ particle that corresponds to the equations (3) or (5), and (4) or (6), respectively. $\mathrm{m}^{\mathrm{i}}$ corresponds to the mass of an individual particle that corresponds to the mass of the bottom of the same equations.

\section{RESULTS}

\section{SEM and TEM Results}

Illite SEM images (figure 2a, left) show that particles are arranged in spherical agglomerates. This arrangement results probably from water evaporation during the drying process. As a consequence, it was difficult to determine the size of the small particles of illite by SEM. On the contrary TEM images show, that particle sizes are homogenous around $40 \mathrm{~nm}$, with regular hexagonal basements (Figure 2a, right) as expected for well-crystallized illites. Typical morphologies of kaolinite particles are shown in Figure $2 \mathrm{~b}$ and 2c, which clearly evidence the heterogeneity in particle sizes. As for illite pseudo hexagonal basements are obtained and this is also the expected shape for crystallized kaolinites [29]. Typical particle sizes can be estimated from SEM and TEM images and are given there only for indication because of the size heterogeneity and the low number of measured particles. Sizes measured from SEM range between 500 and $350 \mathrm{~nm}$ for Kga2 and between 620 and $480 \mathrm{~nm}$ for Khg. Indicative averaged lengths, obtained from TEM on 10 particles, are very similar for the two samples, around $435 \mathrm{~nm}$.

\section{Low-pressure argon adsorption results}


Figure 3 shows the derivatives of argon adsorption isotherms with their DIS decomposition. DIS modeling parameters are collected in Table 1.

As usually observed for argon adsorption on phyllosilicates, the three derivative curves present common features $[4,16,19,30]$ : a peak at medium energy, corresponding to the adsorption on basal faces, a shoulder and a tail at high energy (left hand side of the derivatives) assigned to the adsorption on edge faces and an exponential increase at low energy corresponding to low energy adsorption sites and multilayer adsorption. The decomposition of the derivative adsorption isotherms reveals the following trends:

For illite, 5 adsorption domains are required to model the experimental curve $[18,19,20]$. The three high energy domains, with a major peak located at $\ln \left(\mathrm{P} / \mathrm{P}_{0}\right)$ around -6.95 , are assigned to the adsorption on edge faces. The two low energy domains with a major peak located at $\ln \left(\mathrm{P} / \mathrm{P}_{0}\right)$ values around -4.3 correspond to the adsorption on basal faces $[19,20]$. Taking into account a 13.8 $\AA^{2}$ cross sectional area [31] for adsorbed argon, the surface areas derived from adsorbed amounts on corresponding domains are $38 \mathrm{~m}^{2} / \mathrm{g}$ and $133 \mathrm{~m}^{2} / \mathrm{g}$ for edge and basal faces, respectively.

The derivatives obtained for kaolinite samples exhibit the same features: the broad peak corresponding to the adsorption on basal faces is centered on -3.90 and is modeled with two domains (1 and 2). The basal specific surface areas derived from the two domains are 17.7 and $14.5 \mathrm{~m}^{2} / \mathrm{g}$ for Kga2 and Khg, respectively. The high-energy part, corresponding to the adsorption on edge faces, is modeled with 3 domains (domain 3 to 5) and calculated surface areas are 3.0 and $2.4 \mathrm{~m}^{2} / \mathrm{g}$ for Kga2 and Khg, respectively.

To estimate the mean particle dimensions of illite and kaolinite, the particles were modeled as perfect regular plates with hexagonal basements. Using particle density $\rho$ and the basal and edge surface areas, the thickness (h) and length (l) of the particles were calculated using the following equations: 
$h=2 / \rho . B S A$

$l=8 / \sqrt{3} \cdot \rho \cdot E S A$

The calculated thickness are small for illite particles, $6 \mathrm{~nm}$, and large for kaolinite particles, 43 and $53 \mathrm{~nm}$ for Kga2 and Khg, respectively. Mean lengths are 40, 508 and $636 \mathrm{~nm}$ for illite, Kga2 and Khg, respectively and are in good agreement with the information derived from SEM and TEM micrographs.

\section{AFM results}

Previous studies demonstrated the relevance of the AFM investigation to quantify the geometrical features of individual particles. From the AFM images, the geometrical features of each particle could be extracted by using the two methods described below (see materials and methods). 147, 257 and 154 particle profiles where analyzed for illite, Kga2 and Khg, respectively (Figure 4), without deconvolution procedure to remove tip-sample interaction artifacts in order to minimize the number of calculation steps [2] and to check for the feasibility to derive specific surface areas from raw AFM data. In addition, no particle selection was performed to respect as soon as possible the statistical particle size distribution of the sample.

The respective histograms for individual particles of total specific surface area (Figure 5) for the two calculation methods demonstrated slight differences following the consideration of whole or half of the particle. The three histograms present relatively narrow lognormal distributions for the three samples (Figure 5). The comparison of maximum modes observed on these distributions with the weight-averaged total specific surface area indicated on the figure by open and filled circles revealed the heterogeneity in sizes of the analyzed particles This comparison shows that Kgh sample is the most heterogeneous one. In fact, such variation is induced by the sensitivity of the method in the number of slice considered in the calculation; in our case approximately ten slices were considered. 


\section{Discussion}

The use of argon as molecular probe allows to determine adsorption energy distributions which can be used to derive geometrical properties, specific surface area and main face distribution, of crystallized solids [19,20,21]. In the case of Khg, AFM TSA derived from half or whole particle calculation methods are systematically overestimated when compared to argon (Table 2). As observed by TEM and SEM this probably results from the broad particle size distribution of this sample, which prevents AFM imaging in the whole size range as suggested by the AFM surface area distribution of figure 5. In the present case the imaging technique was in favor of small particles, and thus of high specific surface areas. As a consequence, mean particles sizes derived from the two techniques are also different (Table 3).

The AFM results for illite and Kga2 compare much better with argon adsorption results (Table 2) whatever the calculation method (half or whole particle). Argon TSA of illite, ranges between half and whole particle AFM total surface areas. For Kga2 the AFM results are only slightly lower than argon result. For these two samples, mean sizes derived from both techniques are also in good agreement (Table 3). As already demonstrated for kaolinites, this show that AFM can be used to estimate specific surface areas of very small clay particles as it is the case for illite. However, calculated specific surface areas do not allow choosing for the appropriate AFM calculation method. The comparison of geometrical indexes can be used for that purpose. Indeed, for the illite and Kga2 it is possible to obtain AFM ESA/TSA ratios consistent with the values measured by argon adsorption. In that case, the reliable calculation method is determined without ambiguity: half particle method for illite and total particle procedure for the kaolinite. It can be pointed out that in the case of Kga2, the best calculation method to derive ESA/TSA gives also the closest argon and AFM TSA. In addition, the obtained 0.14 ratio is also in good agreement with the results of Sutheimer et al. [29], Zhou et al. [32] and Bickmore et al. [12] who measured 170, 170 and 77 Kga2 particles, respectively. In the three cases, reported ESA/TSA where 0.18, 
calculated with whole particle method without tip deconvolution by Sutheimer et al. [29] and Zhou et al. [32] and the half particle method with tip deconvolution by Bickmore et al. [12]. The mean height of Kga2 determined to 41nm by Sutheimer et al. [29] and Zhou et al. [32] is also in good agreement with our results obtained with argon and AFM whole particle method (Table 3). Although the total specific surface area of Khg was overestimated, its ESA/TSA ratio was interestingly reliable by using, as for Kga2, the whole particle method.

The fact that comparable ESA/TSA can be obtained from argon and AFM for Khg suggests that particle shape does not depend so munch on particle size distribution, i.e. that the shape of big Khg particles, not analyzed by AFM, is close to the shape of the small Khg particles. The independence between particle size and particle shape was analyzed for the three samples by plotting basal surface area of each particle as a function of its total surface area, by considering geometrical surface areas which favors big particles (Figure 6 left) and specific surface areas which favors small particles (Figure 6 right). On the obtained figures, linear relationships are obtained in each case. For illite and Kga2, calculated slopes corresponding to BSA/TSA are identical when big or small particles are favored. This means that for these two samples, particle shape can be considered as constant, whatever the particle size. This is not the case for Khg as different slopes are obtained for small and big particles. The mean AFM BSA/TSA and argon adsorption BSA/TSA range between the two calculated Khg slopes of figure 6. It can be concluded that ESA/TSA of big particles, not analyzed by AFM, is probably higher than 0.17 (as derived from the slop on fig 6c). As similar BSA/TSA (or ESA/TSA) argon and AFM values are obtained for this sample, big Khg particles contribute significantly to the sample mass but only slightly to edge and basal surface areas.

\section{Conclusion}


The present study shows that excellent agreement can be obtained between the routine AFM and argon adsorption determination of total, basal and edge surface areas of non swelling clay minerals. For samples having broad particle size distribution argon adsorption is more accurate than AFM because of the huge amount of particles in the adsorption system: in the present study, around $1.910^{15}$ and $1.210^{13}$ and $2.510^{14}$ particles for illite, Kga2 and Khg, respectively. The advantage of AFM is that it enables to obtain distributions of surface areas and other parameters and informs on particle size and surface heterogeneities. In addition, AFM is more popular and accessible than low pressure gas adsorption. It seems then useful to propose guidelines for usable and accurate implementation of the AFM approach to derive geometrical properties of clay minerals. Concerning the calculation method, the present study confirms that tip deconvolution that is time consuming is not obligatory. The main question concerns the choice between half and whole particle calculation methods. It seems that whole particle method should be used for big particles as it is the case in other studies with kaolinite. For small particles such as the studied illite half method is the recommended method. Whatever the particle size heterogeneity, accurate AFM TSA is not critical to derive accurate aspect ratio. This aspect ratio can be estimated by considering the slope between total and basal surface area (as in figure 6a, b, c). The comparison with total and basal specific surface areas (Figure 6d, e, f) provide also a good indication on the dependence between particle size and particle shape. The AFM specific surface area can be independently validated and precised by using conventional gas adsorption setups (nitrogen BET) which are much more popular and easy to find than low pressure quasi-equilibrium gas adsorption devices.

Acknowledgment: the authors thank INTAS European program (00-505 project) and GDR PARIS for financial support. 


\section{References Cited}

[1] C. Tournassat, A. Neaman, F. Villiéras, D. Bosbach, L. Charlet, Amer. Mineral. 88 (2003) 1989.

[2] M.C. Jodin, F. Gaboriaud, B. Humbert, Am. Mineral. 89 (2004) 1456.

[3] J.M. Cases, P. Cunin, Y. Grillet, C. Poinsignon, J. Yvon, Clay Miner. 21 (1986) 55.

[4] J.M. Cases, F. Villiéras, L. J. Michot, C.R. Acad. Sci., Paris, Sci. Terre Plan. 331 (2000) 763.

[5] K.L. Nagy, in: K.L. Nagy, A.E. Blum (Eds.), Scanning probe microscopy of clay minerals, The Clay Mineral Society, Boulder CO, 1994, p. 204.

[6] G. Kollensperger, G. Friedbacher, A. Krammer, Fresen. J. Anal. Chem. 363 (1999) 323.

[7] C.M. Eggleston, High-resolution scanning probe microscopy: tip-surface interaction, artifacts, and applications in mineralogy and geochemistry, CMS Workshop Lectures, vol.7, Scanning Probe Microscopy of Clay Minerals, 1994, p. 1.

[8] C.M. Eggleston, G. Jordan, Geochim. Cosmochim. Acta 62 (1998) 1919.

[9] F. Gaboriaud, J.J. Ehrhardt, Geochimi. Cosmochim. Acta 67 (2002) 967.

[10] B. Prelot, F. Villieras, M. Pelletier, G. Gerard, F. Gaboriaud, J.J. Ehrhardt, J. Perrone, M. Fedoroff, J. Jeanjean, G. Lefevre, L. Mazerolles, J.L. Pastol, J.C. Rouchaud, C. Lindecker, J. Colloid Interface Sci. 261 (2003) 244.

[11] M. Zbick, R.S.C. Smart, in: H. Kodama, A. Mermut, J. Torrance (Eds.), Clays for our Future. Proceedings of the International Clay Conference, Ottawa, Canada, 1997, p. 361.

[12] B.R. Bickmore, K.L. Nagy, P.E. Sandlin, T.S. Crater, Am. Mineral. 87 (2002) 780.

[13] E. Balnois, S. Durand-Vidal, P. Levitz, Langmuir 19 (2003) 6633.

[14] M. Zbik, R.S.C. Smart, Clays Clay Miner. 46 (1998) 153.

[15] O. Lietard, J. Yvon, J.F. Delon, R. Mercier, J.M. Cases, In: P. Somasundaran (eds.), in: Fin particles Proccessing, vol. 1, New York, 1980, p. 558.

[16] F. Villiéras, J.M. Cases, M. François, L.J. Michot, F. Thomas, Langmuir 8 (1992) 1104.

[17] F. Villiéras, L.J. Michot, F. Bardot, J.M. Cases, M. François, W. Rudzinski, Langmuir 13 (1997) 1104.

[18] F. Villiéras, L.J. Michot, F. Bardot, M. Chamerois, C. Eypert-Blaison, M. François, G. Gérard, J.M. Cases, C. R. Geosciences 334 (2002) 597-609.

[19] F. Bardot, Les minéraux argileux et leur hétérogénéité superficielle : Influence de la nature des cations compensateurs de l'illite sur les mécanismes d'adsorption de gaz, Thèse de Doctroat en Géosciences, Institut National Polytechnique de Lorraine Nancy, 1998.

[20] F. Bardot, F. Villiéras, L.J. Michot, M. François, G. Gérard, J.M. Cases, J. Dispersion Sci. Technol. 19 (1998) 739.

[21] L.J. Michot, F. Villiéras, Clay Miner. 37 (2002) 39.

[22] C. Eypert-Blaison, F. Villiéras L.J. Michot, M. Pelletier, B. Humbert, J. Ghanbaja, J. Yvon, Clay Miner. 37 (2002) 531.

[23] B. Prélot, F. Villiéras, M. Pelletier, A. Razafitianamaharavo, F. Thomas, C. Poinsignon, J. Colloid Interface Sci. 264 (2003)343.

[24] Z. Zhou, W.D. Gunter, Clays Clay Miner. 40 (1992) 365.

[25] P.M. Costanzo, S. Guggenheim, Clays Clay Miner. 49 (2001) 371.

[26] L.J. Michot, M. François, J.M. Cases, Langmuir 6 (1990) 667.

[27] F. Villiéras, L.J. Michot, J.M. Cases, I. Berend, F. Bardot, M. François, G. G., J.Yvon, in: W. Rudzinski, w.A. Steele and g. Zgrablich (Eds.), Equilibria and Dynamics of Gas Adsorption on Heterogeneous Solid Surfaces, in: Studies Surface in Surface Science and Catalysis, vol. 104, Elsevier Science, Amesterdam, 1997, p. 573.

[28] B.R. Bickmore, E. Rufe, S. Barrett, M.F.J. Hochella, Am. Mineral. 88 (2003) 709.

[29] S.H. Sutheimer, P.A. Maurice, Q. Zhou, Am. Mineral. 84 (1999) 620. 
[30] L.J. Michot, F. Villieras, M. François, J. Yvon, R. Le Dred, J.M. Cases, Langmuir 10 (1994) 3765.

[31] A.L. Mcclellan, H.F. Harnsberger, J. Colloid Interface Sci. 23 (1967) 577.

[32] Q. Zhou, Surface characteristics and dissolution kinetics of two standard kaolinites, Master’s, Kent State University, 1996. 


\section{Figure Caption}

Fig.1. Cartoon of stacked phyllosilicate layers.

Fig.2. Morphology of (a) Illite, (b) Kga2 and (c) Khg samples studied from SEM: left and TEM: right micrographs.

Fig. 3. Experimental derivative Ar adsorption obtained at $77 \mathrm{~K}$ on Na-clays: a) Illite [20, 21], b) Kga2 and c) Khg, with their decomposition using the Derivative Isotherm Summation (DIS) method and BET-Hill model.

Fig. 4. Selected AFM particles cutout in different slices along the Z- profiles: a) illite b) Kga2 and c) Khg.

Fig. 5. Frequency histograms for specific surface area of a) illite, b) Kga2 and c) Khg. White bars: half particle calculation method, Black bars: whole particle calculation method. Circles correspond to weight averaged specific surface areas.

Fig. 6. Comparison between basal and total surface areas of illite (a and d), Kga2 (b and e) and Khg (c and f), expressed in terms of particle geometrical areas (a, b and c) or particle specific surface areas (d, e and f) by using half particle calculation method (illite) and whole particle calculation method (kaolinites).

\section{Table Caption}

Table 1. Main parameters obtained from the application of the DIS method (BET-Hill model) to Ar adsorption at $77 \mathrm{~K}$ on Na-clays: illite [20, 21], Kga2 and Khg.

Table 2. Morphological parameters of the clays obtained by low-pressure argon Adsorption and AFM. ${ }^{*} E S A=100 \times(1-a)$ with a: slope of the linear distribution between basal surface and total surface.

Table 3. Height and basal length of clay particles obtained by low-pressure argon adsorption and AFM measurements. 


\begin{tabular}{ccccc}
\hline & & Illite & Kga2 & Khg \\
\hline Domain 1 & $\ln \left(\mathrm{P} / \mathrm{P}_{0}\right)$ & -2.66 & -2.16 & -2.55 \\
& $\mathrm{Vm}\left(\mathrm{cm}^{3} / \mathrm{g}\right)$ & 16.55 & 1.02 & 0.88 \\
& $\mathrm{w} / \mathrm{kt}^{2}$ & 0 & 0 & 0 \\
& $\mathrm{TSA}\left(\mathrm{m}^{2} / \mathrm{g}\right)$ & 61.4 & 3.8 & 3.3 \\
\hline Domain 2 & $\ln \left(\mathrm{P} / \mathrm{P}_{0}\right)$ & -4.53 & -3.84 & -3.93 \\
& $\mathrm{Vm}\left(\mathrm{cm}^{3} / \mathrm{g}\right)$ & 19.19 & 3.74 & 3.03 \\
& $\mathrm{w} / \mathrm{kt}^{2}$ & 1.7 & 1.3 & 1.2 \\
& $\mathrm{TSA}\left(\mathrm{m}^{2} / \mathrm{g}\right)$ & 71.2 & 13.9 & 11.2 \\
\hline Domain 3 & $\ln \left(\mathrm{P} / \mathrm{P}_{0}\right)$ & -6.95 & -6.78 & -6.81 \\
& $\mathrm{Vm}\left(\mathrm{cm}^{3} / \mathrm{g}\right)$ & 8.00 & 0.61 & 0.55 \\
& $\mathrm{w} / \mathrm{kt}^{2}$ & 0.5 & -0.8 & -0.8 \\
& $\mathrm{TSA}\left(\mathrm{m}^{2} / \mathrm{g}\right)$ & 29.7 & 2.3 & 2.1 \\
\hline Domain 4 & $\ln \left(\mathrm{P} / \mathrm{P}_{0}\right)$ & -10.15 & -9.93 & -9.90 \\
& $\mathrm{Vm}\left(\mathrm{cm}^{3} / \mathrm{g}\right)$ & 2.03 & 0.13 & 0.04 \\
& $\mathrm{w} / \mathrm{kt}^{2}$ & 0 & 0 & 0 \\
& $\mathrm{TSA}\left(\mathrm{m}^{2} / \mathrm{g}\right)$ & 7.5 & 0.5 & 0.2 \\
\hline Domain 5 & $\mathrm{Ln}\left(\mathrm{P} / \mathrm{P}_{0}\right)$ & -13.03 & -12.16 & -11.88 \\
& $\mathrm{Vm}\left(\mathrm{cm}^{3} / \mathrm{g}\right)$ & 0.21 & 0.07 & 0.04 \\
& $\mathrm{w} / \mathrm{kt}^{2}$ & 0.5 & 0 & 0 \\
& $\mathrm{TSA}\left(\mathrm{m}^{2} / \mathrm{g}\right)$ & 0.7 & 0.3 & 0.1 \\
\hline
\end{tabular}

Table 1. Main parameters obtained from the application of the DIS method to Ar adsorption at 77 K on Na-clays: illite, Kga2 and Khg.

\begin{tabular}{|c|c|c|c|c|c|}
\hline \multicolumn{3}{|c|}{ No. particles } & Ar Adsorption & AFM total particle & AFM half particle \\
\hline \multirow{4}{*}{ Illite } & 147 & ESA & $38(22 \%)$ & $20.6(11 \%)$ & $29.9(20 \%)$ \\
\hline & & ESA* & & $11 \%$ & $19 \%$ \\
\hline & & BSA & 133 & 165 & 120.7 \\
\hline & & TSA & 171 & 185.6 & 150.6 \\
\hline \multirow[t]{4}{*}{ Kga2 } & 257 & ESA & 3.0 (15\%) & $2.3(14 \%)$ & $2.3(28 \%)$ \\
\hline & & ESA* & & $13 \%$ & $26 \%$ \\
\hline & & BSA & 17.7 & 14.2 & 9.8 \\
\hline & & TSA & 20.6 & 16.5 & 13.6 \\
\hline \multirow[t]{4}{*}{ Khg } & 154 & ESA & $2.4(14 \%)$ & 7.3 (14\%) & $9.2(22 \%)$ \\
\hline & & ESA* & & $17 \%$ & $24(\%)$ \\
\hline & & BSA & 14.5 & 43 & 32.6 \\
\hline & & TSA & 16.9 & 50.3 & 41.8 \\
\hline
\end{tabular}

Table 2. Morphological parameters of the clays obtained by low pressure argon Adsorption and AFM. *ESA $=(1-a) * 100$ with a: slope of the linear distribution between basal surface and total surface. 


\begin{tabular}{ccccc}
\hline & & Illite & Kga2 & Khg \\
\hline AFM mean & Height (nm) & $6 \pm 5$ & $36 \pm 54$ & $11 \pm 24$ \\
& Average Length (nm) & $55 \pm 27$ & $504 \pm 484$ & $155 \pm 169$ \\
\hline Ar Adsorption & Height (nm) & 5.7 & 43.1 & 52.7 \\
& Basal Length (nm) & 46.4 & 587.7 & 734.7 \\
& & & & \\
\hline
\end{tabular}

Table3. Height and basal length of clay particles obtained by Argon Adsorption and microscopy measurements AFM. 


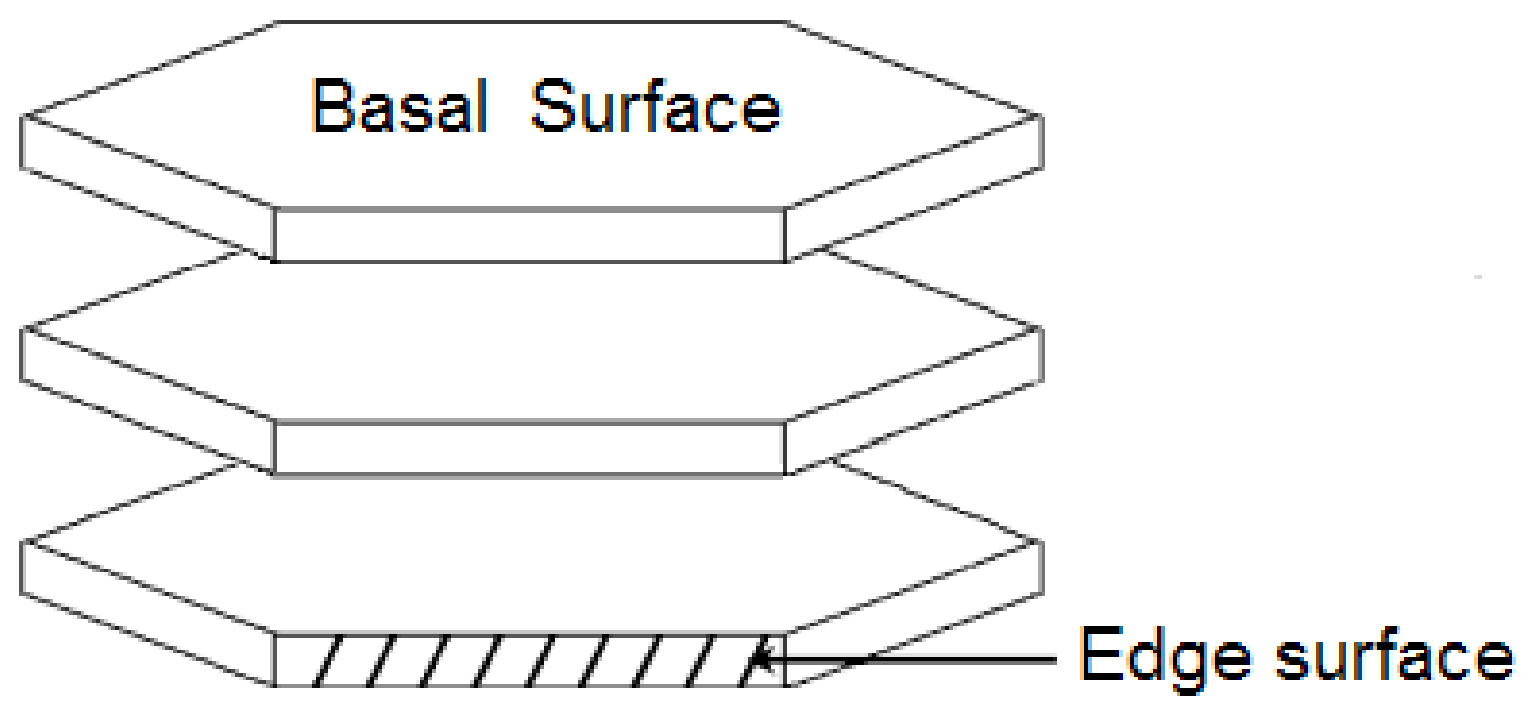

Fig.1. Cartoon of stacked phyllosilicate layers. 
a)

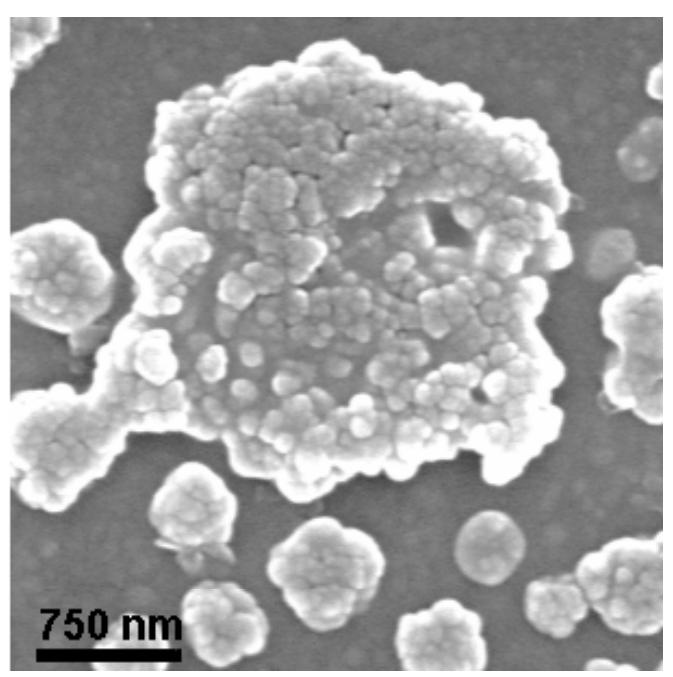

b)

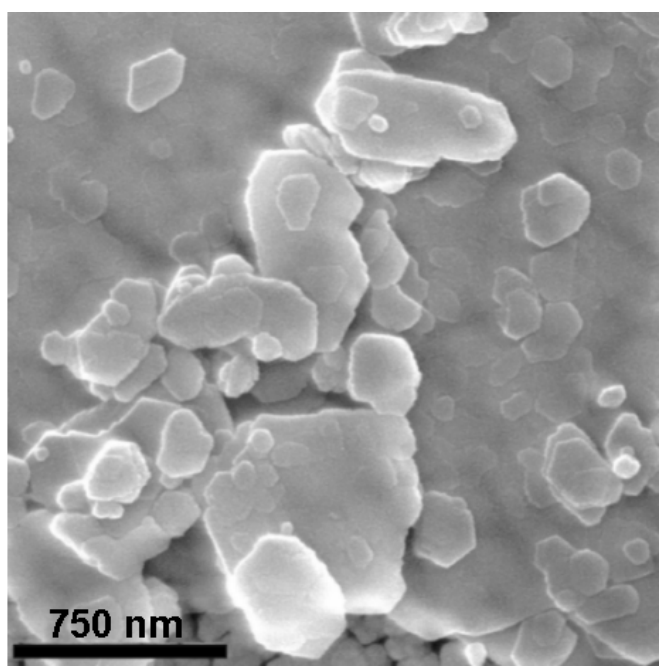

c)

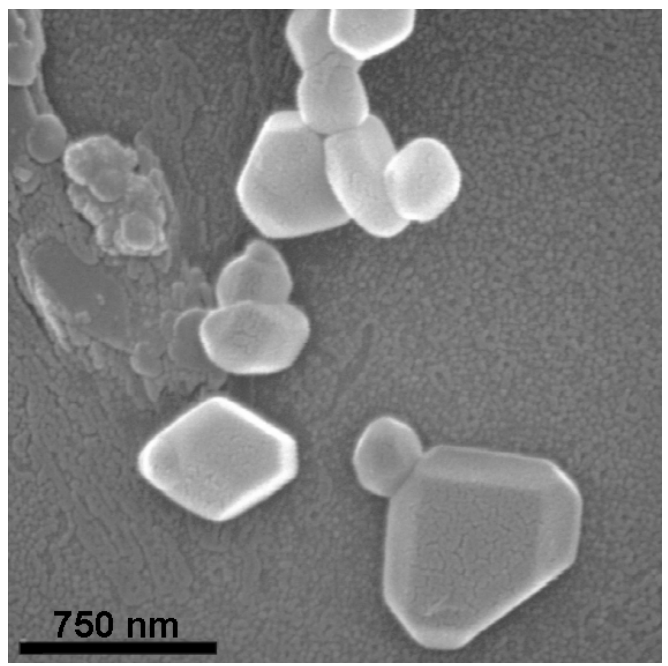

J. Colloid Interface Sci. 296 (2006) 614-623
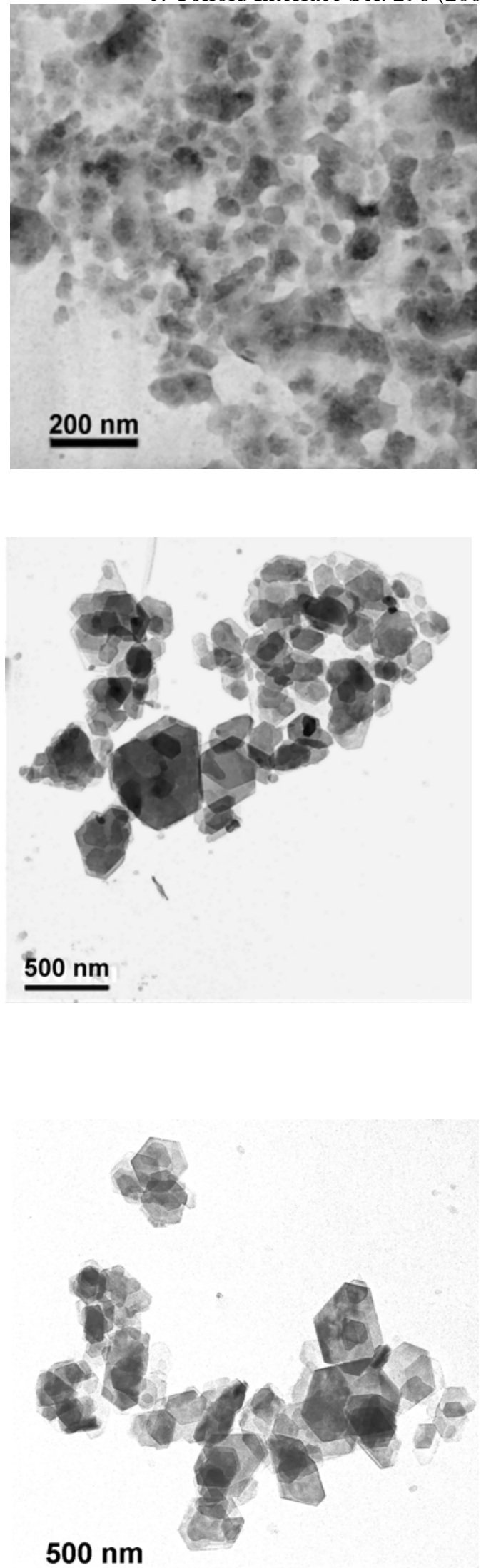

Fig.2. Morphology of (a) Illite, (b) Kga2 and (c) Khg samples studied from SEM: left and TEM: right micrographs. 

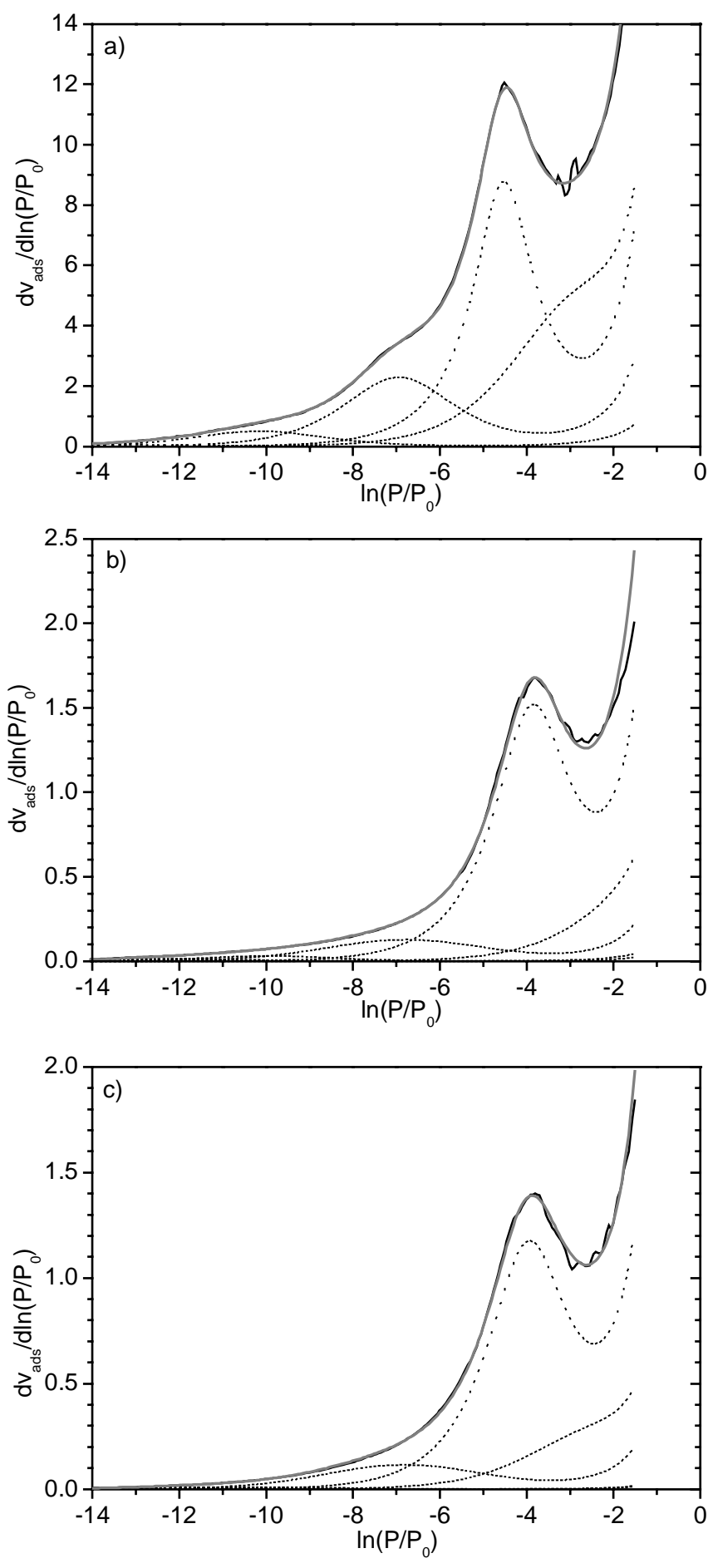

Fig. 3. Experimental derivative Ar adsorption obtained at $77 \mathrm{~K}$ on Na-clays: a) Illite [20, 21], b) Kga2 and c) Khg, with their decomposition using the Derivative Isotherm Summation (DIS) method and BET-Hill model. 

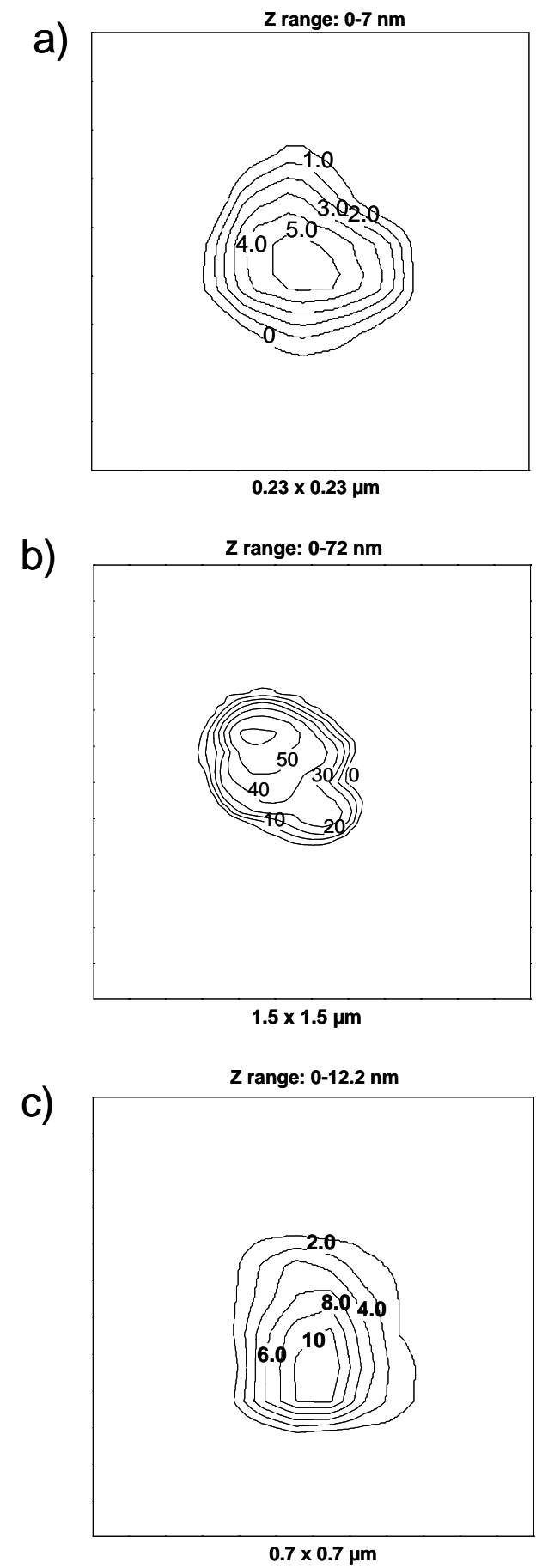

Fig. 4. Selected AFM particles cutout in different slices along the Z- profiles of: a) illite b) Kga2 and c) Khg. 

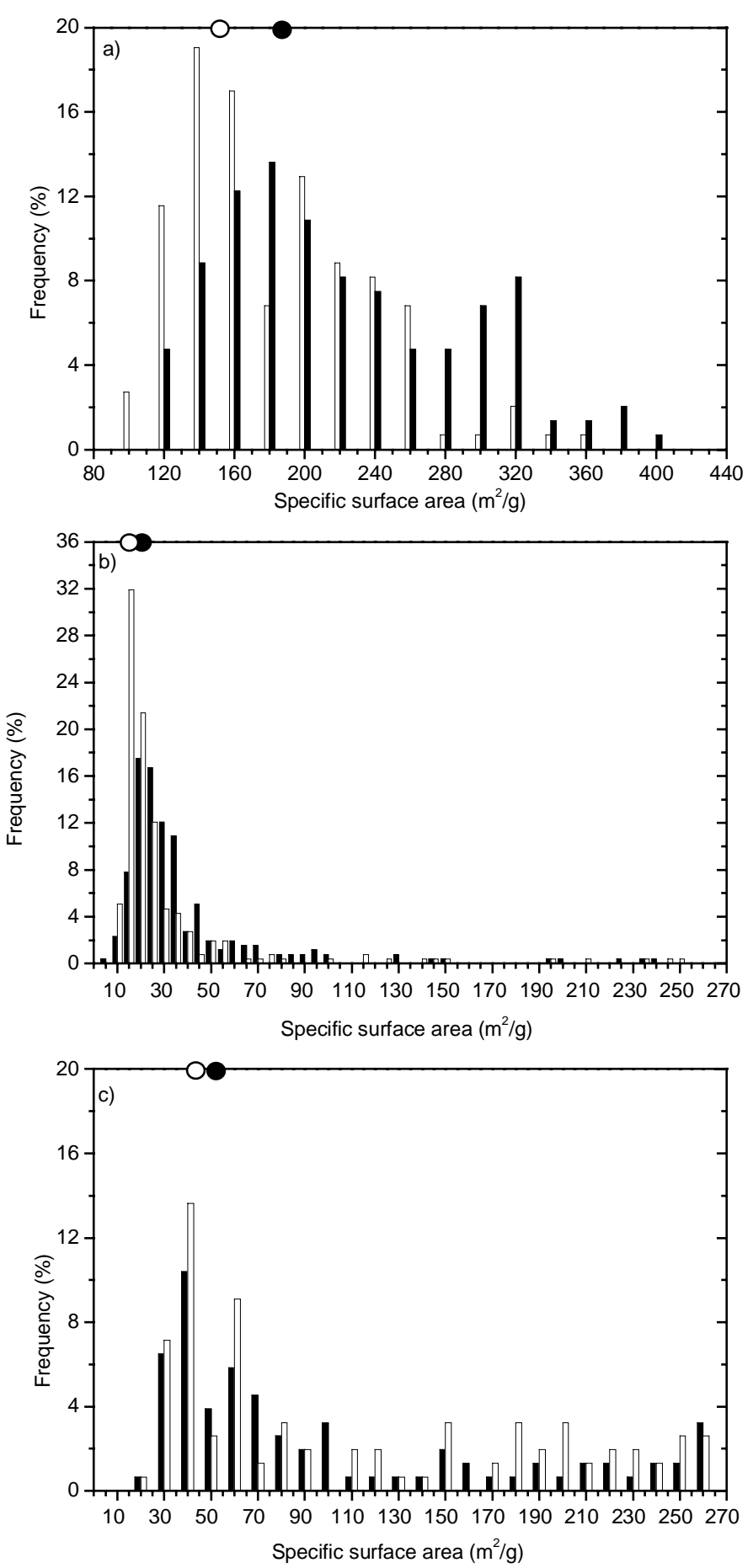

Fig. 5. Frequency histograms for specific surface area of a) illite, b) Kga2 and c) Khg. White bars: half particle calculation method, Black bars: whole particle calculation method. Circles correspond to weight averaged specific surface areas. 

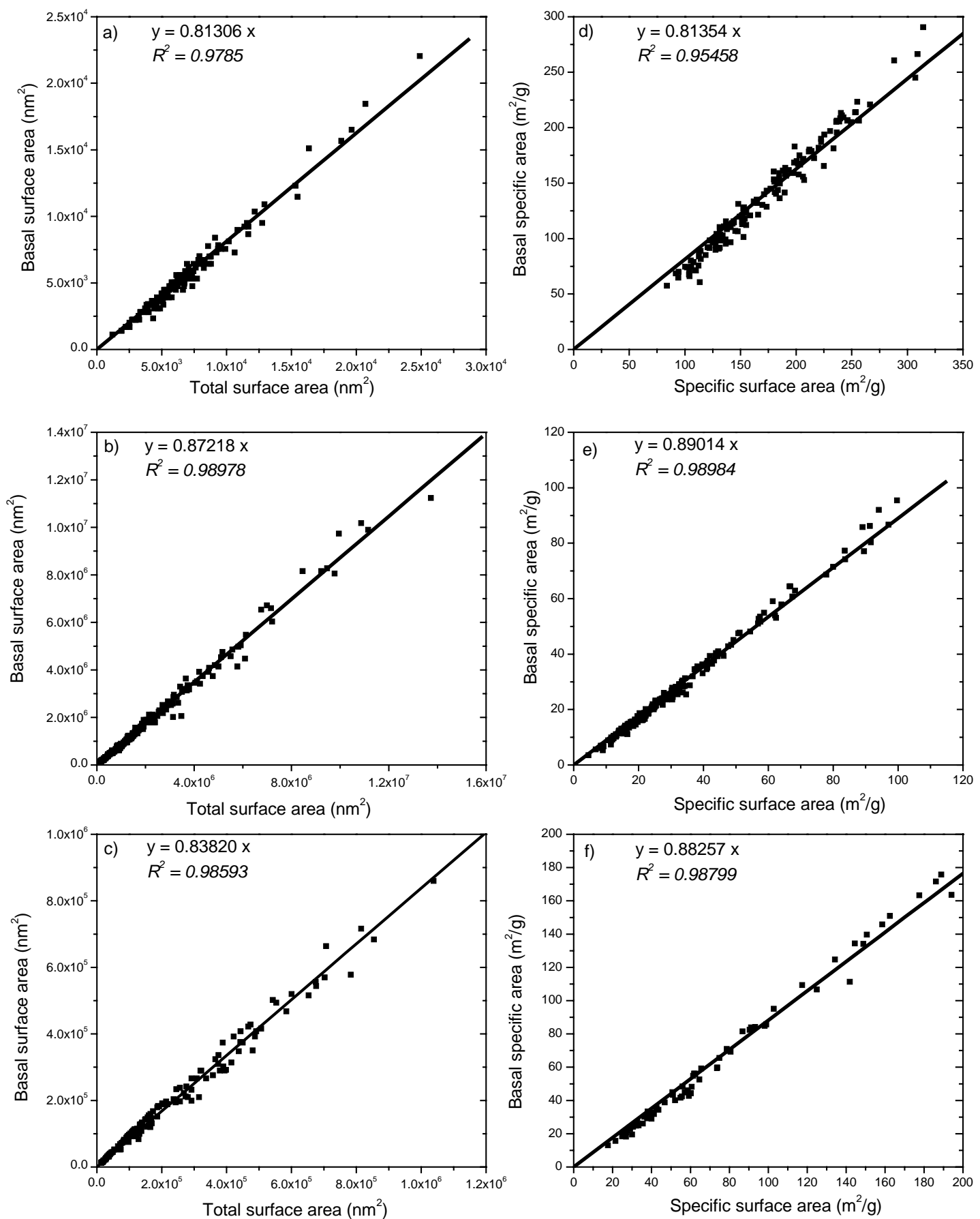

Fig. 6. Comparison between basal and total surface areas of illite (a and d), Kga2 (b and e) and Khg (c and $\mathrm{f}$ ), expressed in terms of particle geometrical areas (a, b and c) or particle specific surface areas (d, e and f) by using half particle calculation method (illite) and whole particle calculation method (kaolinites). 